Paper:

\title{
Decimation-Free Directional Filter Banks for Classification and Numbering on Posterior Dental Radiography Using Mesiodistal Neck Detection
}

\author{
Agus Zainal Arifin*, Anny Yuniarti*, Wijayanti Nurul Khotimah*, \\ Arya Yudhi Wijaya*, Ahmad Mustofa Hadi*, Naser Jawas ${ }^{* *}$, and Eha Renwi Astuti*** \\ * Department of Informatics, Institut Teknologi Sepuluh Nopember (ITS) \\ Kampus ITS, Surabaya, 60111, Indonesia \\ E-mail: \{agusza, anny, wijayanti, arya\} @if.its.ac.id \\ ** Sekolah Tinggi Manajemen Informatika dan Teknik Komputer (STIKOM) \\ Bali, Denpasar, Indonesia \\ E-mail: naser.jawas@gmail.com \\ *** Department of Dental Radiography, Airlangga University \\ Surabaya, Indonesia \\ E-mail: e_renwi_a@yahoo.com \\ [Received October 2, 2013; accepted March 24, 2014]
}

\begin{abstract}
Dental classification and numbering on posterior dental radiography are important tasks for forensic and biomedical applications. This paper proposed a novel method of classification and numbering on posterior dental radiography using Decimation-Free Directional Filter Bank (DDFB) and mesiodistal neck detection. The method was started by a segmentation method for decomposing dental image into directional images using DDFB. Detection of mesiodistal neck tooth separated the crown and the root of teeth. Finally we used support vector machine for classification and numbering. The experimental results achieved a classification accuracy rate of $91 \%$. It approved the robustness of the proposed method for solving the problem of dental classification and numbering.
\end{abstract}

Keywords: mesiodistal neck, dental radiography, decimation-free directional filter banks, dental classification, dental numbering

\section{Introduction}

One of the important aspects in the forensic radiology is dental classification. This classification is used for identifying people using postmortem radiological images of teeth. Teeth can be invaluable in forensic, because in many cases teeth are the only distinctive remains to survive.

Moreover, identifying using dents is more reliable in several cases, for example, in tsunami, natural disaster or even traffic accident. The above mentioned give serious defect to the victim's body, so it's too difficult to analyze using another parts of the human body. In those cases, human body usually gets serious damages, except some strong parts like the teeth. Therefore, teeth are chosen as the identification factor to help the forensic investigation.

The first step in an automated identification system includes teeth classification. The teeth are determined by its type and number. Every tooth has its own information, thus tooth classification is an important phase to extract information for identification purposes.

The problem of dental classification has been identified and solved by some researchers. Reference [1] has presented an approach to classify and number the teeth bitewing image. They used Bayesian classification to distinguish molars and premolars teeth and number with respect to the international standard numbering of teeth. Fourier descriptor of dental bitewing image is used to be the feature of the classification model.

This approach has its own success until it meets the limits in several cases. It fails to classify some teeth which have no root part in their image. Using the Fourier descriptor of contour also has its consequence, that is, the condition of crown of both molar and premolar can be so identical. Thus it is difficult to distinguish them using this feature. This error has a big impact in the final step, numbering, and it can decrease the total accuracy of the classification system.

In order to overcome the previous problem, the algorithm of [2] is offered. It provides a binary linear Support Vector Machine (SVM) to replace the Bayesian and uses length-width ratio as the feature. This algorithm performs well except for some conditions that we cannot rely based on the length-width ratio of several images, due to the broken teeth, uncompleted image, i.e. without root, or even the problem of differentiation of root and gums. In [3], Mesiodistal neck is used to be the feature of the classification model due to its stability in age raising and teeth broken. However the robustness in various type of dental image is not considered. Indeed, the segmentation for 
separating each tooth is a difficult task due to the unevenly illumination, low contrast, and domination of dark color on dental radiography. Less quality of segmentation leads to less result accuracy of classification.

Since we are only interested in distinguishing between molars and premolars, we need to obtain a clear outline of each object. The teeth segment is mainly in vertical direction, while the gum is in horizontal direction. Therefore, a method for classification and numbering which uses direction-based approach for extracting each tooth in a proper shape is needed.

In this paper, we proposed a method for decomposing dental image into directional images using DecimationFree Directional Filter Bank (DDFB) and classifying and numbering on posterior dental radiography using SVM with mesiodistal neck detection. It facilitates forensic odontologists to search through large numbers of dental records.

Details of this approach will be provided in the next section. We will explain the literature review, detailed proposed method, and brief explication of each step, followed by the experimental result, and data analysis and conclusion.

\section{Literature Review}

\subsection{Decimation-Free Directional Filter Bank}

Directional Filter Bank (DFB) was originally introduced by Bamberger and Smith in 1992 [4]. DFB contains a set of wedge-shaped frequency filters that can be used to decompose image into directional components. Bamberger DFB also uses a decimation process which can make the result maximally decimated but still allows the original image to be exactly reconstructed.

In 2005, Khan, M.A.U. et al. introduced new DFB without decimation process. It is called Decimation-Free DFB (DDFB) [5]. The decimation process from Bamberger DFB is used to obtain a compression effect which result uses interpolation in its process. The interpolation can lead to false artifacts in the output image. Therefore, it is not suitable for an image enhancement process $[5,6]$. Details of comparisons between DFB and DDFB can be found in [5].

DDFB design process, like Bamberger DFB, is separated into phases. In the first phase, it only needs 2 filters. These are $H_{00}\left(\omega_{1}, \omega_{2}\right)$ and $H_{11}\left(\omega_{1}, \omega_{2}\right)$ or horizontal and vertical filter. The filters consist of an hourglass-shape as shown in Fig. 1. The output image has the same size as the input image because no down-sampler is applied during the process in the first phase [6].

The second phase of DDFB build 4 different filters. The second phase filters are derived from the first phase filters. The process can be continued to make $2^{n}$ filters from $n$-phases. An example of the second phase filters are shown in Fig 2.

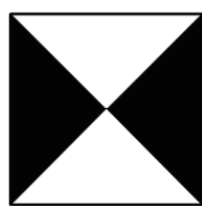

(a)

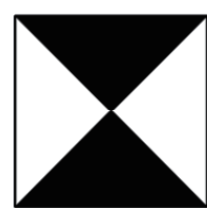

(b)
Fig. 1. Hourglass-shaped passband filters from first phase DDFB.

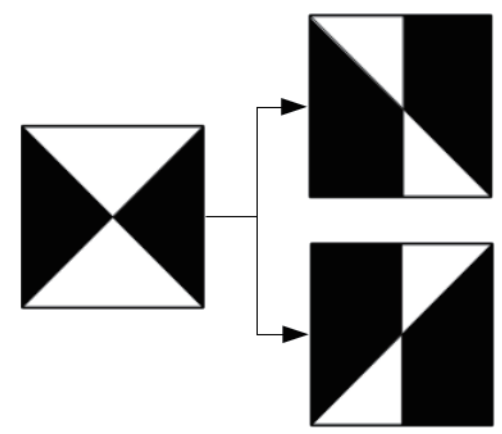

(a)

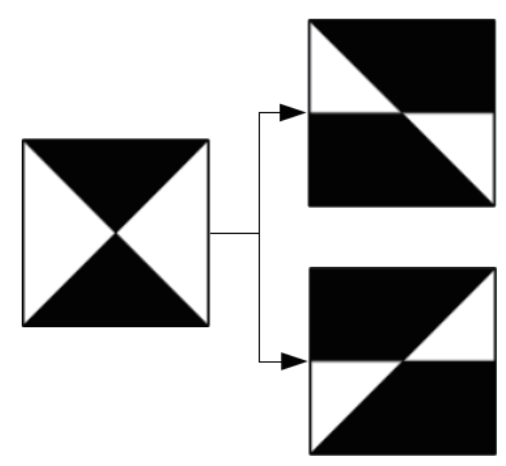

(b)

Fig. 2. Second phase DDFB filters.

\subsection{Homomorphic Filtering}

Homomorphic filtering process can be used to normalize non-uniform illumination in image [6]. The processes are described in [7] as follows. First, the natural logarithm of input image $i(x, y)$ is taken by calculating

$$
z(x, y)=\ln (i(x, y)) \text {. }
$$

Then, the frequency domain of $z(x, y)$ is formed by using Fourier transform:

$$
Z(u, v)=F\{z(x, y)\},
$$

where $i(x, y)$ is the input image with $x$ width and $y$ height. $Z(u, v)$ is the output fourier. The $x$ and $y$ represent index of 2 dimensional matrix image in spatial domain whereas $u$ and $v$ represent index of 2 dimensional matrix spectrum in frequency domain. 


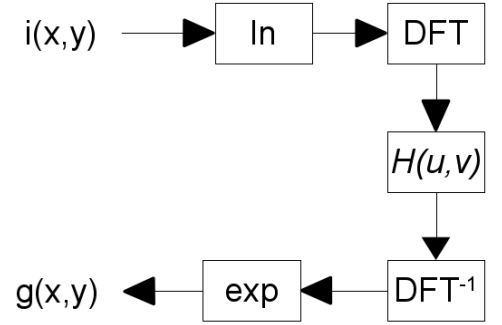

Fig. 3. Homomorphic filtering process.

Now, $Z(u, v)$ can be filtered using frequency filter $H(u, v)$ by

$$
S(u, v)=Z(u, v) H(u, v) .
$$

The filtered spectrum frequency $S(u, v)$ is transformed back into the spatial domain using an inverse Fourier Transform

$$
S(x, y)=F^{-1}\{S(u, v)\} .
$$

In the last step, because $z(x, y)$ is formed by natural logarithm, exponential of $s(x, y)$ is needed to reverse the natural logarithm to get the output image

$$
g(x, y)=e^{s(x, y)} .
$$

The complete sequence of homomorphic filtering process is shown in Fig. 3.

\section{Proposed Method}

Our proposed method can be divided into 3 main modules. The first module is dental image segmentation. The second module is feature extraction and the last module is classification and numbering.

The first module contains series of preprocessing methods for preparing the input image for feature extraction process in the second module. Processes in the first module are image enhancement using DDFB and Homomorphic filtering, image binarization and teeth separation.

We use DDFB to separate the horizontal image and vertical image. After horizontal and vertical sections of the image are separated into directional images, we can apply different homomorphic filtering parameters to control the enhancement of each directional image. We use separated vertical and horizontal image for an enhancement because teeth image data contain many edges in horizontal and vertical direction. The edges between each tooth are a combination of vertical edge of tooth and horizontal edge of gum. We need different enhancement parameters to strengthening vertical edge from each tooth and softening horizontal edge of gum. With different enhancement parameters, we can obtain better separation for each tooth. Homomorphic filtering reduces non-uniform intensity in each directional image; therefore it also smoothly reduces the edges. After each directional image is enhanced using homomorphic filtering, we combine both directional images into one image. The combined image is used as enhanced image and then it is binarized. The binarized image is used to distinguish between teeth (foreground) and other object in the image (background).

The second module is the feature extraction module. The feature extraction is using binarized image as input. The Mesiodistal Neck areas are detected using integral projection and connected component labeling. The last module is the classification and numbering module. Classification process is done by using Support Vector Machines (SVM). The complete flowchart of our proposed method is as shown in Fig. 4.

\subsection{Dental Image Segmentation}

Segmentation process is one of the most important aspects which determines the result of the later process. The purpose of this module is to separate each tooth in the input image from non-tooth regions such as gums, background and other undesired regions. The better the segmentation result, the better the classification result obtained.

There are several processes in the segmentation phase. Firstly, a dental input image is decomposed using the $1^{\text {st }}$ phase DDFB to obtain horizontal and vertical images. The process of DDFB decomposition is as follows. Input image is transformed into frequency domain using $2 \mathrm{D}$ Fourier Transformation. The spectrum frequency from the 2D Fourier Transformation is filtered using 2D passband filters from DDFB as shown in Fig. 1. The filtered spectrums are transformed back into spatial domain using 2D Inverse Fourier Transformation. The output images are 2 directional images, horizontal image and vertical images. Fig. 5 shows an example of input image and directional images from the $1^{\text {st }}$-phase DDFB process.

The next process is to reduce the non-uniform illumination in directional images by using Homomorphic filtering. In this research, Gaussian highpass filter is used inside the homomorphic filtering. A Gaussian spread parameter is utilized as the parameter of homomorphic filtering. This parameter determines the spread area of Gaussian filters. The higher the spread parameter set, the more normalized non-uniform illumination image produced. The effect of Gaussian highpass filter in images is an edge sharpening in the result image.

Homomorphic filtering process is applied to both the directional images but with a different parameter. The parameter is manually set to get the desired result.

After Homomorphic filtering process, the horizontal and vertical images are recombined into one image to form an enhanced input image in the Directional Image Unification process. The recombination process is as follows

$$
D I U=\text { horizontalImage }-(1-n * \text { verticalImage }),
$$

where $n$ is a manual input parameter to find the best binarization result. In this research, we use $n=1$ to 3 . The horizontalImage and verticalImage have pixel values between 0 and 1. Fig. 6 shows the sample result of the homomorphic filtering 


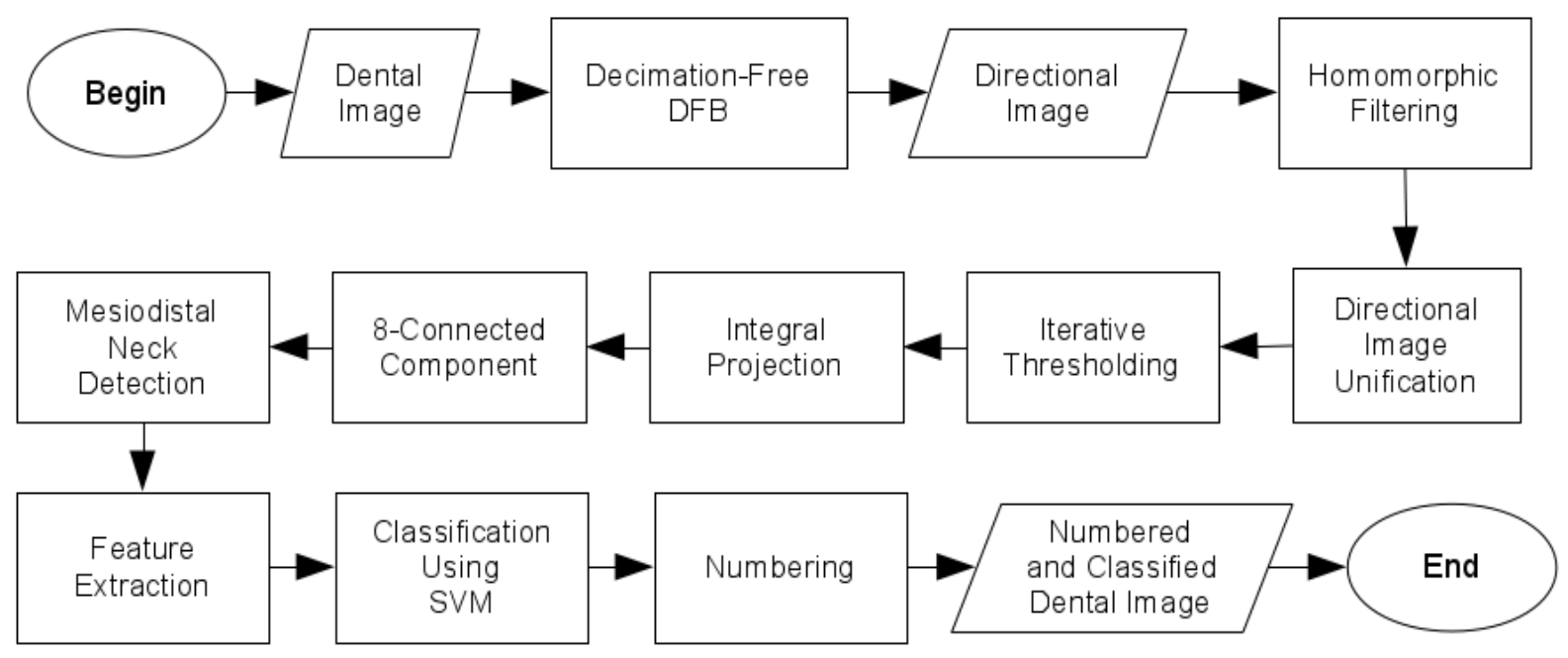

Fig. 4. The proposed method.

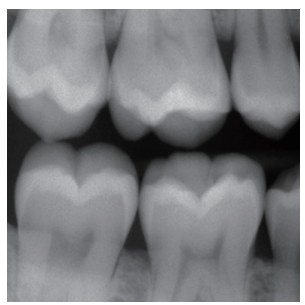

(a)

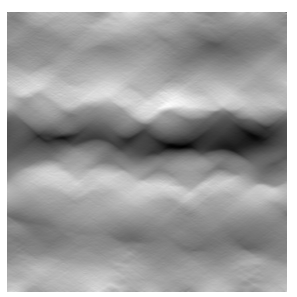

(a)

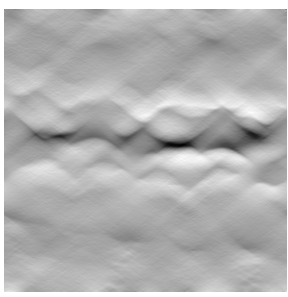

(b)

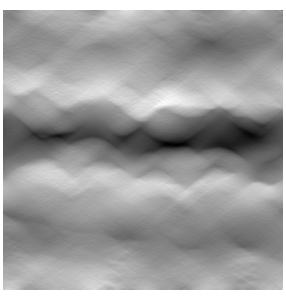

(b)

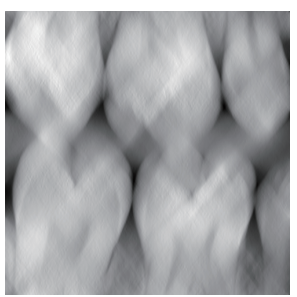

(c)

Fig. 5. (a) Input image, (b) Horizontal image, and (c) Vertical image.

\subsection{Image Binarization}

This phase is a step to transform the grey level image into a binary image. This is really important for the teeth region to be distinguished from image background, gum, and all undesired regions.

An adaptive thresholding is chosen to achieve the objectives of binarization. In addition, some morphological operations have been done, e.g. closing and opening to minimize the number of noise during this phase. Fig. 7 shows the result of Directional Image Unification process and its binarization. The binary image is generated with Otsu's thresholding method [8] to show the result of separation of teeth from the other region.

Fig. 6. Comparison of Images: (a) Before the homomorphic filtering and (b) After the homomorphic filtering.

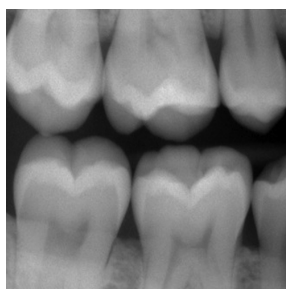

(a)

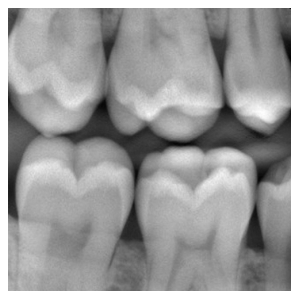

(b)

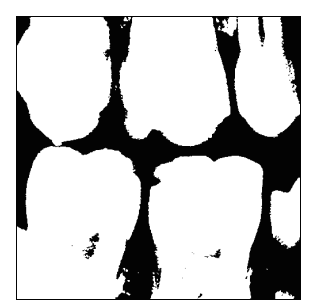

(c)
Fig. 7. (a) Original input, (b) Directional image unification result and (c) Binarization result. 


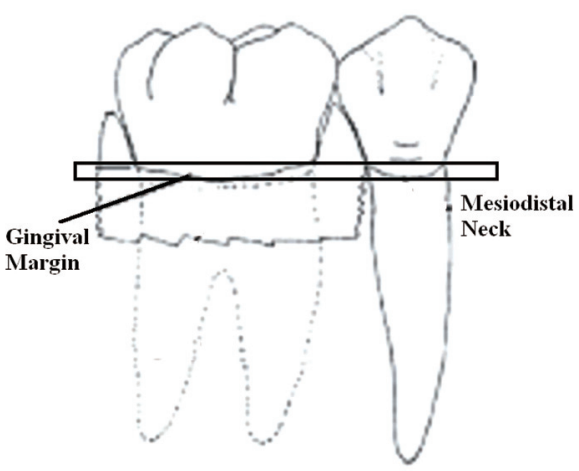

Fig. 8. The area of mesiodistal neck [3].

\subsection{Teeth Separation}

Teeth separation is one step to choose the ROI of the tooth. It separates the maxilla and mandible using the algorithm of horizontal projection, and it separates each tooth using the vertical projection. The eight-connected components labeling is also used to create small regions that consist only each single tooth.

\subsection{Dental Image Classification}

The main objective of this phase is to design a classification model using Support Vector Machines (SVM) for assigning teeth in the class where they should belong and each of their numbers. This paper proposes a feature called Mesiodistal Neck to support the accuracy of classification.

Before explaining this method, it is necessary to understand the contribution of Mesiodistal neck teeth. A mesiodistal neck tooth is a line between cementum and enamel, which separates the crown and root of tooth. Several studies use crown only, whereas some teeth has similar form of crown. In that case, the crown can be the only variable to depend on. Also in some cases, the tooth root cannot be figured out, because it is blurring and the boundary between the root and the gums is not clear. Mesiodistal neck is shown in the Fig. 8.

The mesiodistal neck can be found by a minimum width of tooth between crown and root. We can see from Fig. 8 that mesiodistal neck is shown by the area near the gum. The tooth area near gum is shown to have minimum width.

\subsection{Proposed Mesiodistal Neck Teeth Detection}

The tooth neck is a place where the crown has its narrow width and the root has its wider width. In a normal tooth, the width is stable within $1 / 3$ from the base of the root.

Normal teeth usually show an image which stand $90^{\circ}$, so that we can easily detect the spot of the neck. But in many cases, teeth are not shown vertically. They create an angle with the $x$-axis. In order to achieve the best extraction of the teeth neck, a rotation is needed before the width counting.

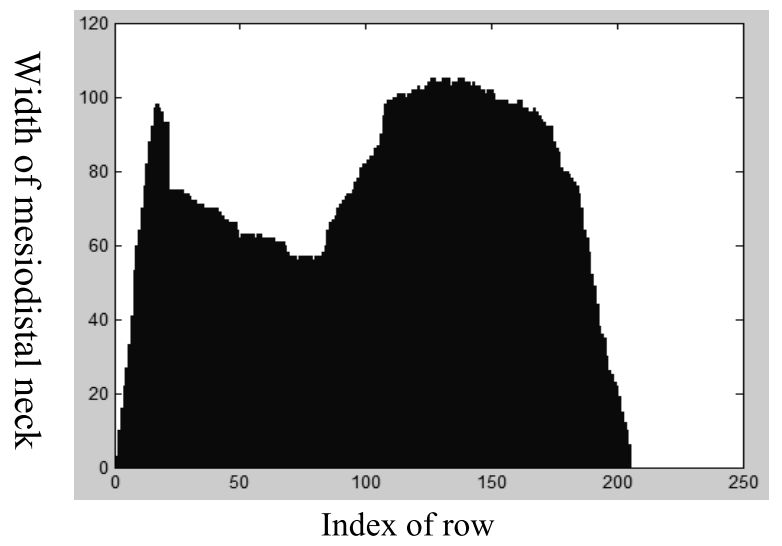

Fig. 9. Histogram representation of mesiodistal neck width.

First we have to count the skew angle of the tooth $(\alpha)$, and then represent this angle into a degree between $-90^{\circ}$ and $90^{\circ}$. In case of making a vertical tooth, we can rotate the object by $-90^{\circ}$ or $90^{\circ}$. If $\alpha<0$, we can rotate the object by $-90^{\circ}$ to make the object vertical. Otherwise, if $\alpha>0$, we choose the $90^{\circ}$ rotation. This approach enables us to count the width of the tooth more precisely.

After the teeth have been rotated, the width of each tooth can be counted using Eq. (7) and a group of width can also be selected as the mesiodistal.

$$
\text { Mesiodistal }[i]=\max \left(y_{i}\right)-\min \left(y_{i}\right),
$$

where $i$ is the order of row in the tooth contour, maximum and minimum column in the row $i$-th, shown by $\operatorname{Max}\left(y_{i}\right)$ and Min $\left(y_{i}\right)$. The row can be used as height of tooth and column is the width of tooth.

The group of those widths in Mesiodistal Neck can be represented in a histogram. The characteristic of Mesiodistal neck stated in the previous section is the base to search which point can be chosen as Neck. In the histogram, in Fig. 9, it is shown that there are some points that can be stated as the extreme point. The mesiodistal neck is found when the previous point has a decreasing pattern of width and it shows a stable pattern. It's the point between the crown and root, which most narrow. We can represent it in an iteration approach, so the iteration will terminate when it found its divergence point. The found width is then used to be the mesiodistal neck value and its position is saved as the neck line of the tooth. After recording the value of mesiodistal neck of each tooth in the current object, the classification can be started next.

\subsection{Classification}

We used supervised learning for classifying the type of teeth into molars or premolars. Support Vector Machines (SVM) is chosen because of its robustness and simplicity. The basic idea of SVM is to transform data into higher dimensional space and find the optimal hyperplane with maximal separation margin between classes.

Data is assumed as linearly separable and will be proven in the next phase. The hyperplane of our binary 
SVM has a general form as follow: $w \circ x+b=0$, where $w$ is the normal to the hyperplane and $\frac{b}{\|w\|}$ is the perpendicular distance from the hyperplane to the origin.

We train our SVM using training data to have an optimal classifier system. Moreover, we used the system to run our testing data to know its performance. Each vector of testing data has to be evaluated, and assigned each tooth as molar or premolar with respect to the $y^{\prime}$ function, where $y^{\prime}=\operatorname{sgn}\left(w^{*} \circ x^{\prime}+b^{*}\right)$ represents the vector function, sgn is the sign function, and $x^{\prime}$ is the test data. Each data will belong to molar if $y^{\prime}>0$, otherwise it will be premolar.

The input data for this classification process is the tooth area and the width of mesiodistal. The mesiodistal neck tooth is measured by searching the minimum number of consecutive white pixel in each row of binarized tooth image. The area of tooth is measured by the total number of pixel in each binarized tooth. In this research, we use linear kernel for the SVM because the data tend to be Linear.

\subsection{Teeth Numbering}

If all teeth have been classified as Molars or Premolars, then we need to assign a particular number to each tooth. In teeth numbering we assign each teeth with a unique number according to the universal teeth numbering system $(1-32)$. The classification results are formatted in a pattern of " $\mathrm{P}$ " or " $\mathrm{M}$ " which correspond to premolars and molars, respectively, and indicate the pattern of the current image.

We saved the default pattern of posterior teeth both in maxilla and mandible in the arrangement corresponding to the groundtruth. The input pattern for this phase should follow the sequence of the default format. Obtaining a match sequence format helps us when assigning the number of each tooth.

We need to find a pattern which matches the pattern which has been classified in the previous step. In fact, the groundtruth shows all teeth number for each pattern. Therefore, we need to find the most similar part of the groundtruth with this pattern. Finally, we will have a specific number of each tooth according to the universal teeth numbering system.

\section{Experimental Results}

The proposed method is implemented using MATLAB and LibSVM. The input data consists of 15 and 10 images which constitute 110 and 67 teeth for both the training and testing data, respectively.

\subsection{Segmentation}

Dental segmentation results show good performance. The accuracy is $94 \%$ with 63 teeth recognized out of 67. In the failure cases, the tooth graylevel values where closer to the graylevel value of the gums and background as shown in Figs. 10(a) and (b). The tooth in bottom left is

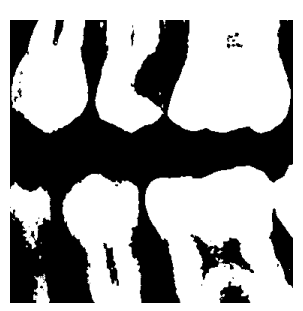

(a)

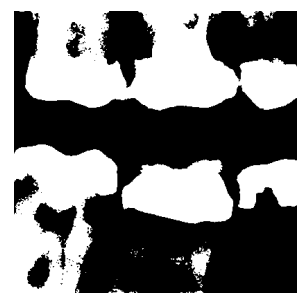

(c)

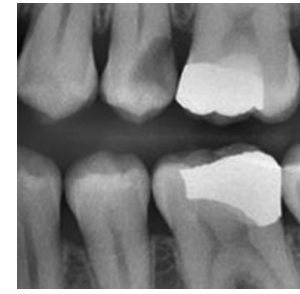

(b)

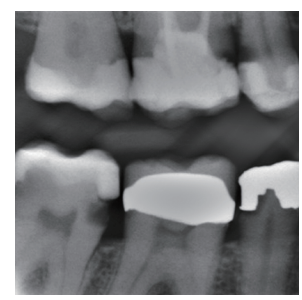

(d)
Fig. 10. Example of failure segmentation result.

Table 1. Classification accuracy.

\begin{tabular}{cccccc}
\hline No. & Image & \multicolumn{4}{c}{ Classification Result } \\
\cline { 3 - 6 } & Samples & TP & FP & FN & TN \\
\hline 1 & A & 4 & 0 & 0 & 1 \\
2 & B & 3 & 0 & 0 & 3 \\
3 & C & 3 & 0 & 1 & 1 \\
4 & D & 4 & 0 & 0 & 1 \\
5 & E & 2 & 1 & 0 & 3 \\
6 & F & 3 & 2 & 1 & 0 \\
7 & G & 5 & 1 & 0 & 3 \\
8 & H & 5 & 0 & 0 & 4 \\
9 & I & 4 & 0 & 0 & 4 \\
10 & J & 4 & 0 & 0 & 4 \\
\hline
\end{tabular}

not recognized properly. The dental image segmentation process still has a problem of separating the teeth from the non-teeth regions, when the teeth regions has gray level values close to non-teeth regions. Another failure case happens when the tooth contains dental filling material as shown in Figs. 10(c) and (d). The dental filling materials appear brighter than the other region of teeth. This causes other parts of the tooth not recognized.

\subsection{Classification}

The SVM classifies a tooth to be recognized as molar or premolar. The True Positive (TP) class is increased when a molar tooth is classified as molar tooth. The True Negative (TN) class is increased when a premolar tooth is classified as premolar. The False Positive (FP) and False Negative (FN) are increased when misclassified between molar and premolar.

The complete classification result is given in Table 1. It shows the number of TP, FP, FN, and TN from two classes (molar and premolar). The classification result 
Table 2. Numbering accuracy.

\begin{tabular}{ccccc}
\hline No. & Image & \multicolumn{2}{c}{ Numbering Result } & Not \\
\cline { 3 - 4 } & Samples & True & False & Recognized \\
\hline 1 & A & 3 & 2 & 0 \\
2 & B & 6 & 0 & 0 \\
3 & C & 3 & 2 & 0 \\
4 & D & 3 & 2 & 0 \\
5 & E & 5 & 0 & 1 \\
6 & F & 0 & 4 & 2 \\
7 & G & 8 & 0 & 1 \\
8 & H & 9 & 0 & 0 \\
9 & I & 8 & 0 & 0 \\
10 & J & 8 & 0 & 0 \\
\hline
\end{tabular}

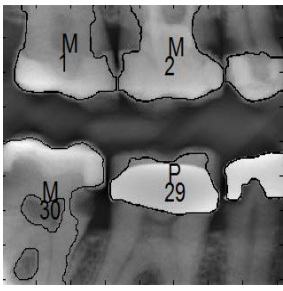

(a)

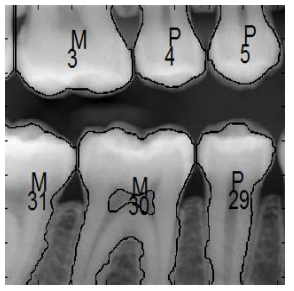

(b)
Fig. 11. Output from the system.

shows good performance with $91 \%$ accuracy. The proposed method can successfully classify 61 of 67 teeth. However four teeth were not recognized due to segmentation failure.

\subsection{Teeth Numbering}

The teeth numbering process also achieved a good result with $84 \%$ accuracy. The false numbering usually happens when the number of a tooth is shifted 1 or 2 from the true tooth number. This affected the other teeth in the same row. Table 2 shows the numbering results of each image.

The best result of classification and numbering was achieved from image B where all teeth are recognized, classified and numbered correctly. The worst results were from image F with 2 unrecognized teeth. Four teeth were not correctly numbered and no premolar tooth was correctly classified. The results from image $\mathrm{B}$ and $\mathrm{F}$ are shown in Figs. 11(a) and (b), respectively.

\section{Discussion}

In this research, all vertical images were normalized using homomorphic filtering with the spread parameter assigned between values 1 and 3 . The higher spread parameter, the more uniform illumination of enhanced image we can achieve.

The parameter $n$ from the directional image unification

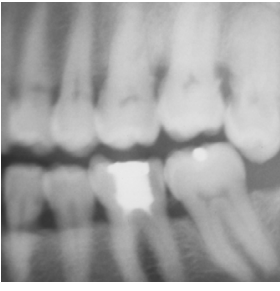

(a)

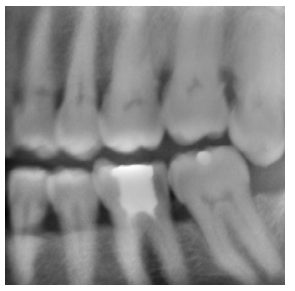

(c) (b)

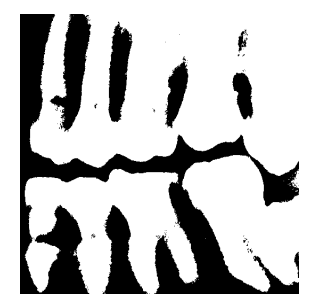

(d)

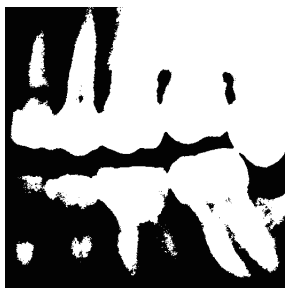

Fig. 12. Example of binarization result from (a) Original image and (b) Binarization of original image and (c) Enhanced image and (d) Binarization of enhanced image.

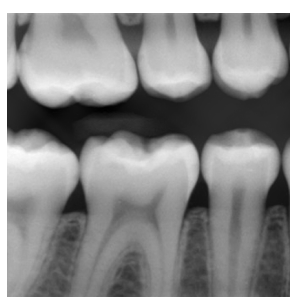

(a)

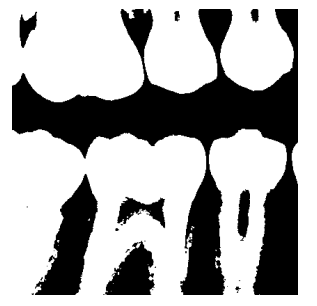

(b)
Fig. 13. Area inside tooth are not recognized as foreground.

process is also set from 1 to 3 . The best result from these two parameters setting is taken as the enhanced image for each original input. The result of binarization from enhanced image shows that the enhancement procedure can help improving the separation of tooth from input image.

It can also help to improve the brightness of the tooth while still preserving uniformity of illumination in the outer area of the tooth as shown in Fig. 12.

Unfortunately, some areas inside the tooth contains darker areas and those areas are still marked as background in the binarization result from enhanced images as shown in Fig. 13.

\section{Conclusion}

Teeth segmentation, classification and numbering still remain important issues of the dental radiographs for forensic research. Automatic identification for disaster victims is absolutely needed due to rapid performance and high accuracy.

In this paper, we proposed a method for decomposing 
dental image into directional images using DecimationFree Directional Filter Bank (DDFB). We also classified and numbered posterior dental radiography using SVM with mesiodistal neck detection.

The enhancement of dental image including the homomorphic filtering and contrast enhancement were introduced in the first phase. This enhancement was followed by the adaptive thresholding for transforming the gray level into binary images. Our main approach in selecting the path of the mesiodistal is also shown in the previous section.

Our algorithm of classification applied achieved high accuracy in various cases. It could overcome the most noise problem, distinguished the image into Premolar and Molar (91\% accuracy) and most teeth were assigned their number precisely. Blur Images with some weak gray level and ambiguous patterns were still needed to be explored for enhancing the performance of the system. Some unexpected cases which have not been found still remained challenges to be solved.

\section{Acknowledgements}

This research was supported by the JICA PREDICT-ITS JointResearch Program Phase 2 and the DGHE (Directorate General of Higher Education), Ministry of National Education and Cultures, Republic of Indonesia.

\section{References:}

[1] M. H. Mahoor and M. Abdel-Mottaleb, "Classification and Numbering of Teeth in Dental Bitewing Images," Pattern Recognition, Vol.38, pp. 577-586, 2005.

[2] P. L. Lin, Y. H. Lai, and P. W. Huang, "An Effective Classification and Numbering System for Dental Bitewing Radiographs Using Teeth Region and Contour Information,' Pattern Recognition, Vol.43, pp. 1380-1392, 2009.

[3] F. Aeini and F. Mahmou, "Classification and Numbering of Posterior Teeth in Bitewing Dental Images," Proc. 3rd Int. Conf. on Advanced Computer Theory and Engineering (CTE), 2010.

[4] R. H. Bamberger and M. J. T. Smith, "A Filter Bank for the Directional Decomposition of Images: Theory and Design," IEEE Trans. on Signal Processing, Vol.40, No.4, pp. 882-893, Apr. 1992.

[5] M. A. U. Khan, M. K. Khan, and M. A. Khan, "Comparative Analysis of Decimation-Free Directional Filter Bank with Directional Filter Bank: In Context of Image Enhancement," Proc. $9^{\text {Th }}$ Int. Multitopic Conf., 2005, pp. 1-8.

[6] P. T. H. Truc, M. A. U. Khan, Y. K. Lee, S. Lee, and T. S. Kim, "Vessel Enhancement Filter using Directional Filter Bank," Computer Vision and Image Understanding, Vol.113, pp. 101-112, 2009.

[7] R. C. Gonzalez and R. E. Woods, "Digital Image Processing $3^{\text {rd }}$ Edition," New Jersey, Pearson Education, Inc. pp. 289-293, 2008.

[8] N. Otsu, "A Threshold Selection Method from Gray-Level Histograms," IEEE Trans. on Systems, Man and Cybernetics, Vol.9, pp. $62-66,1979$.

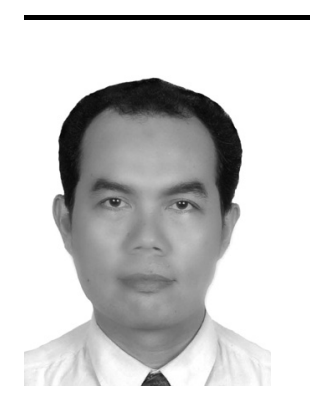

Name:

Agus Zainal Arifin

\section{Affiliation:}

Department of Informatics, Institut Teknologi Sepuluh Nopember (ITS)

\section{Address:}

Kampus ITS, Surabaya, 60111, Indonesia

Brief Biographical History:

1995 Received Computer Bachelor at Sepuluh Nopember Institute of Technology (ITS) Surabaya Indonesia

2001 Received a Master Computer at University of Indonesia

2007 Received the Dr. Degree from the Department of Information

Engineering, Graduate School of Engineering, Hiroshima University,

Japan

2008- An Associate Professor of Informatics Department at ITS Surabaya

Main Works:

- Computer vision, information retrieval

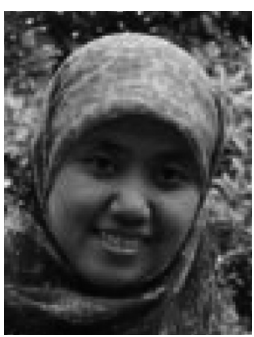

Name:

Anny Yuniarti

\section{Affiliation:}

Department of Informatics, Institut Teknologi Sepuluh Nopember (ITS)

\section{Address:}

Kampus ITS, Surabaya, 60111, Indonesia

Brief Biographical History:

2003 Received her Computer Bachelor at Sepuluh Nopember Institute of Technology Surabaya Indonesia

2005- A Lecturer of Informatics Department at Sepuluh Nopember Institute of Technology Surabaya Indonesia

2008 Received her Master from computer science engineering of

University of Western Australia, Australia

Main Works:

- Image processing, computer vision, computer graphics 


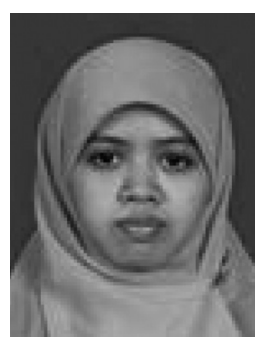

Name:

Wijayanti Nurul Khotimah

\section{Affiliation:}

Department of Informatics, Institut Teknologi Sepuluh Nopember (ITS)

Address:

Kampus ITS, Surabaya, 60111, Indonesia

Brief Biographical History:

2008 Received her Bachelor Degree in Informatics from Institut

Teknologi Sepuluh Nopember (ITS) Surabaya, Indonesia

2011 Received her Master Degree from computer science of National

Taiwan University of Science and Technology Taiwan

2011- Working as Lacturer in informatics department of ITS

Main Works:

- Computer vision, image processing, intelligence system

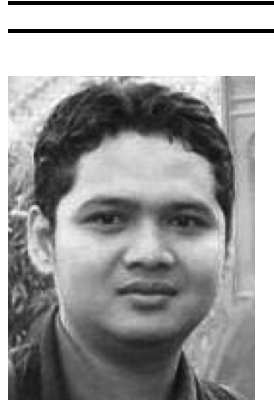

Name:

Arya Yudhi Wijaya

\section{Affiliation:}

Department of Informatics, Institut Teknologi Sepuluh Nopember (ITS)

Address:

Kampus ITS, Surabaya, 60111, Indonesia

Brief Biographical History:

2008 Received Computer Bachelor at Sepuluh Nopember Institute of Technology Surabaya Indonesia

2010 Received Computer Master at Sepuluh Nopember Institute of

Technology Surabaya Indonesia

2010- Lecturer of Informatics Department at Sepuluh Nopember Institute of Technology Surabaya Indonesia

Main Works:

- Image processing, computer vision

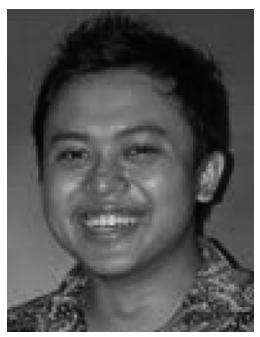

Name:

Ahmad Mustofa Hadi

\section{Affiliation:}

Department of Informatics, Institut Teknologi Sepuluh Nopember (ITS)

\section{Address:}

Kampus ITS, Surabaya, 60111, Indonesia

Brief Biographical History:

2011 Received his Bachelor Degree in Informatics department of ITS,

Indonesia

2013- Studying Ph.D. in Germany

Main Works:

- Image processing, computer graphics

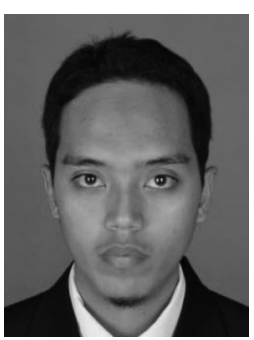

\section{Name:}

Naser Jawas

\section{Affiliation:}

Sekolah Tinggi Manajemen Informatika dan Teknik Komputer (STIKOM)

Address:

Bali, Denpasar, Indonesia

Brief Biographical History:

2009 Received his Bachelor Degree in Electrical Engineering from Universitas Udayana, Indonesia

2013 Earned his Master Degree in Informatics from Institut Teknologi Sepuluh Nopember (ITS) Surabaya, Indonesia

2013- Working as a Lecturer in Department of Computer Engineering, STMIK STIKOM Bali, Indonesia

Main Works:

- Computer vision, image processing, information retrieval

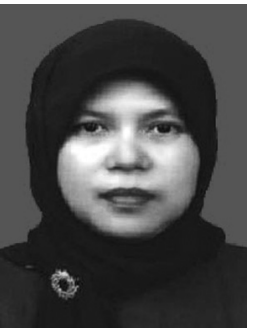

\section{Name:}

Eha Renwi Astuti

\section{Affiliation:}

Department of Dental Radiography, Airlangga University

Address:

Surabaya, Indonesia

Brief Biographical History:

She receives her Bachelor, Master, and Doctoral Degree from faculty of dentistry Airlanggan University. Currently, she is Working as Dentist and Lecturer at faculty of dentistry Airlangga University. 